\title{
Bringing Physiology into PET of the Liver
}

\author{
Susanne Keiding \\ Department of Medicine V (Hepatology and Gastroenterology) and PET Centre, Aarhus University Hospital, Aarhus, Denmark
}

\begin{abstract}
Learning Objectives: On successful completion of this activity, participants should be able to describe (1) the role of the liver's blood supply in the interpretation of dynamic PET measurements for estimating hepatic blood perfusion and metabolism; (2) the limitations of using compartmental analysis of hepatic tracer kinetics in PET studies; and (3) the tracer kinetic advantages of using the metabolic clearance of ${ }^{18} \mathrm{~F}-\mathrm{FDGal}$ in dynamic PET studies of regional metabolic liver function.

Financial Disclosure: The authors of this article have indicated no relevant relationships that could be perceived as a real or apparent conflict of interest.

CME Credit: SNM is accredited by the Accreditation Council for Continuing Medical Education (ACCME) to sponsor continuing education for physicians. SNM designates each JNM continuing education article for a maximum of 1.0 AMA PRA Category 1 Credit. Physicians should claim only credit commensurate with the extent of their participation in the activity.

For CE credit, participants can access this activity through the SNM Web site (http://www.snm.org/ce_online) through March 2013.
\end{abstract}

Several physiologic features make interpretation of PET studies of liver physiology an exciting challenge. As with other organs, hepatic tracer kinetics using PET is quantified by dynamic recording of the liver after the administration of a radioactive tracer, with measurements of time-activity curves in the blood supply. However, the liver receives blood from both the portal vein and the hepatic artery, with the peak of the portal vein time-activity curve being delayed and dispersed compared with that of the hepatic artery. The use of a flow-weighted dual-input time-activity curve is of importance for the estimation of hepatic blood perfusion through initial dynamic PET recording. The portal vein is inaccessible in humans, and methods of estimating the dual-input time-activity curve without portal vein measurements are being developed. Such methods are used to estimate regional hepatic blood perfusion, for example, by means of the initial part of a dynamic ${ }^{18} \mathrm{~F}-\mathrm{FDG} \mathrm{PET} / \mathrm{CT}$ recording. Later, steady-state hepatic metabolism can be assessed using only the arterial input, provided that neither the tracer nor its metabolites are irreversibly trapped in the prehepatic splanchnic area within the acquisition period. This is used in studies of regulation of hepatic metabolism of, for example, ${ }^{18} \mathrm{~F}-\mathrm{FDG}$ and ${ }^{11} \mathrm{C}$-palmitate.

Key Words: PET kinetics; liver PET; liver hemodynamics; liver metabolism; liver function

J Nucl Med 2012; 53:425-433

DOI: 10.2967/jnumed.111.100214

S

everal physiologic features make the interpretation of PET studies of liver physiology and pathophysiology a challenge compared with other organs. The liver is a large organ that weighs around $1.2 \mathrm{~kg}$, has a blood flow of about

Received Oct. 28, 2011; revision accepted Jan. 23, 2012.

For correspondence contact: Susanne Keiding, PET Centre, Aarhus University Hospital, 44 Norrebrogade, DK-8000 Aarhus, Denmark.

E-mail: susanne@pet.auh.dk

Published online Feb. 9, 2012.

COPYRIGHT @ 2012 by the Society of Nuclear Medicine, Inc.
$1.2 \mathrm{~L} / \mathrm{min}$, and plays a key role in the regulation of body metabolic homeostasis and the first-pass metabolism of all substances absorbed from the intestines, including detoxification processes. As illustrated in Figure 1, salient features of importance for PET of the liver are the blood supply from 2 vessels, that is, the hepatic artery (HA; 25\%) and the portal vein (PV; 75\%), a blood volume of 30\%-40\%, microcirculation through highly permeable sinusoids, bile formation, and numerous liver-specific metabolic pathways (1). Patients with acute or chronic liver disease have reduced metabolic functioning, and in patients with chronic liver disease the sinusoidal endothelium is less permeable because of reduced fenestration of endothelial cells and deposits of collagen; that is, the sinusoids have undergone "capillarization." Increased vascular resistance leads to portal hypertension with esophageal varices through which the blood bypasses the hepatic first-pass metabolism of substances absorbed from the blood (1); the accumulation of toxic substances can lead to hepatic encephalopathy and toxic effects on other organs.

As readers of The Journal of Nuclear Medicine will know, the detailed quantification of tracer kinetics using PET is usually performed by kinetic modeling of data from dynamic PET recordings of the organ or tissue examined after the administration of a positron-emitting radioactive tracer with simultaneous measurements of the time course of tracer concentrations in the blood supply. In this review, we will discuss the importance of taking into account the special liver physiology in the interpretation of dynamic liver PET/CT. First, we consider the tracer supply to the liver from both the HA and the PV. Second, interpretation of PET measurements of in vivo biochemistry is based on kinetic modeling of metabolic pathways that have been determined by in vitro and ex vivo studies; the numerous specific metabolic pathways in the liver make the interpretation of PET measurements a challenge in liver studies. In addition, if radiolabeled 

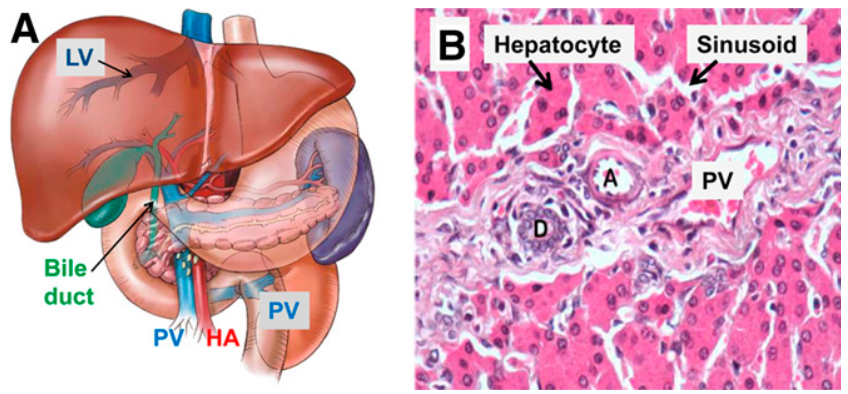

FIGURE 1. (A) Liver is located in upper right part of abdominal cavity. It receives blood from $\mathrm{PV}$, which drains intestines and thereby brings all ingested substances to liver, where they are subjected to first-pass metabolism. Liver also receives arterial blood from HA, which mixes with PV blood before entering specialized capillaries of liver — sinusoids (Fig. 1B) — and empty into liver veins (LV). Liver also produces bile, which is excreted into bile ducts that empty into duodenum. (B) Normal liver histology showing portal triad with branches of PV, branches of HA (A), and bile ducts (D). Blood from PV and HA enters sinusoids lined by hepatocytes, in which metabolism takes place. Blood from sinusoids empties via central veins (not shown) into liver veins (Fig. 1A). Bile flows from hepatocytes via bile canaliculi into bile ducts in opposite direction of blood flow in portal triad.

metabolites formed in the liver, or elsewhere, enter the blood, the blood will contain both unmetabolized tracer and radiolabeled metabolites; this also has to be accounted for in the kinetics modeling. Finally, the interpretation of PET data must incorporate the fact that the PET camera cannot separate radioactive tracers in the vascular and intracellular compartments; this factor is of special importance for PET of the liver because of the large hepatic blood volume.

As is well known, PET/CT is an extremely valuable tool for tumor diagnostics and the assessment of treatment effects, both for primary and for secondary liver tumors (2-5), but this issue is not a subject of the current review. Here, we will discuss the importance of including physiologic aspects in the interpretation of measurements of hepatic blood perfusion and metabolism when examined by dynamic PET. Tracers that have been used in such studies primarily include true tracers, for example ${ }^{15} \mathrm{O}$-carbon monoxide, ${ }^{15} \mathrm{O}$-water, ${ }^{13} \mathrm{~N}$-ammonia, and ${ }^{11} \mathrm{C}$-palmitate, and analog tracers such as the glucose ana$\operatorname{logs}{ }^{18} \mathrm{~F}-\mathrm{FDG}$ and $3-\mathrm{O}^{-1}{ }^{11} \mathrm{C}$-methylglucose, the galactose analog $2-{ }^{18} \mathrm{~F}$-fluoro-2-deoxy-D-galactose $\left({ }^{18} \mathrm{~F}-\mathrm{FDG} a \mathrm{l}\right)$, and the free fatty acid analog $14(R, S)-{ }^{18} \mathrm{~F}$-fluoro-6-thia-heptadecanoic acid.

\section{TRACER SUPPLY FROM THE HA AND PV}

Subsequent to a bolus administration of tracer, the arterial time-activity curve rapidly increases to a peak (Fig. 2, left panel). The HA time-activity curve is identical to that in any other artery, such as the femoral or radial artery, that can be sampled without disturbing the HA flow. For tracers that are distributed throughout the intestines, the peak of the PV time-activity curve is delayed and dispersed compared with that of the HA as a result of passage of the tracer through the prehepatic splanchnic area (Fig. 2: $\left.{ }^{18} \mathrm{~F}-\mathrm{FDG}\right)$. For ${ }^{15} \mathrm{O}$-labeled carbon monoxide, which firmly binds to the erythrocytes, that is, a vascular tracer, the peak of the PV time-activity curve is delayed but not dispersed much (Fig. 2: ${ }^{15} \mathrm{O}$-carbon monoxide) (6). It is apparent from Figure 2 that after approximately 2 min the HA and PV concentrations coincide.

Assessment of the initial distribution of the tracer from the blood to the cells after administration is sensitive to the initial time-activity curves (7), and estimations of the parameters for these processes must accordingly include tracer inputs from both the HA and the PV; the use of only an arterial input will lead to the systematic underestimation of these rate constants $(7-10)$. The flow-weighted dual-in-
FIGURE 2. Examples of time-activity curves in blood supply to liver from HA and PV and dual input. (Left) Time-activity curves measured in femoral artery (red) and PV (blue) after bolus administration of ${ }^{15} \mathrm{O}$-carbon monoxide or ${ }^{18} \mathrm{~F}-\mathrm{FDG}$, respectively, to pigs. Model-derived PV time-activity curves (green) were calculated from arterial time-activity curves using population means of tracer-specific parameters for passage of tracer from intestinal arteries to PV (Eq. 2). (Right) For each tracer, measured dual-input time-activity curve (blue) was calculated from measured arterial time-activity curve and measured PV time-activity curve (blue, left) and individually measured HA blood flow fractions (Eq. 1). Corresponding model-derived dual-input time-activity curves (green) were calculated from measured arterial time-activity curve, model-derived PV time-activity curve, and population mean HA blood flow fraction of 0.25 (Eq. 3). (Reprinted with permission of (6).)

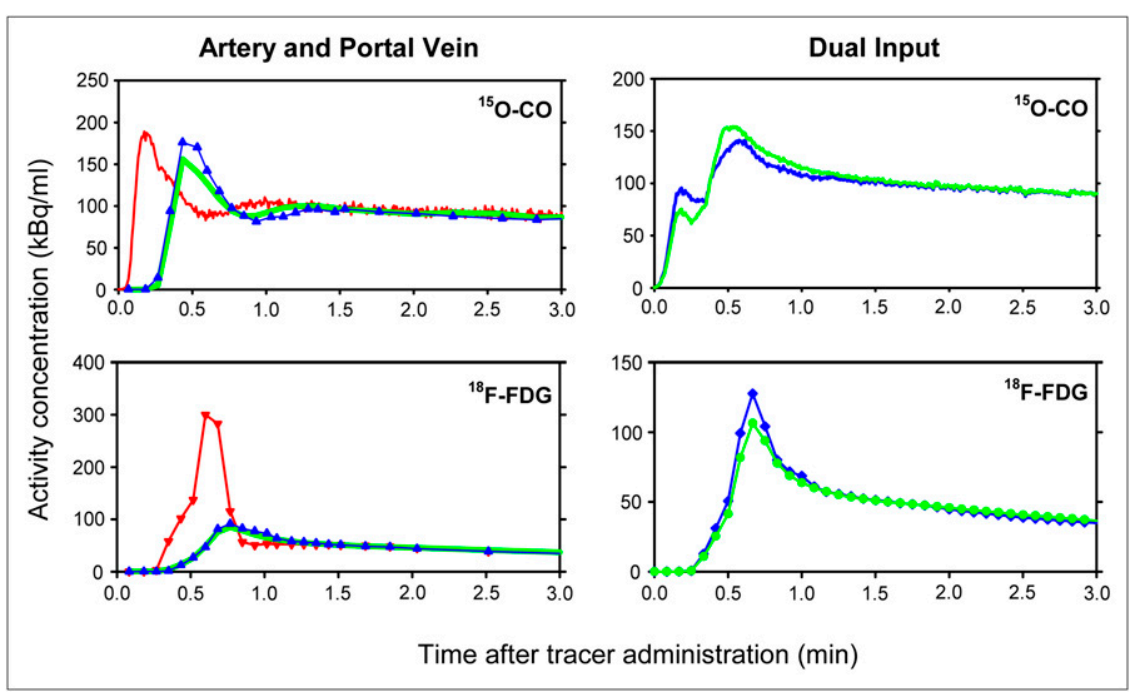


put time-activity curve to the sinusoids can be calculated in pigs by sampling blood from the PV and a peripheral artery combined with flow measurements of the PV and HA:

$$
C_{\text {dual }}(t)=f_{H A} C_{\mathrm{A}}(t)+\left(1-f_{H A}\right) C_{\mathrm{PV}}(t),
$$

where $C_{\mathrm{A}}(t)$ is the HA time-activity curve, $C_{\mathrm{PV}}(t)$ is the PV time-activity curve, $C_{\text {dual }}(t)$ is the dual-input time-activity curve, and $f_{\mathrm{HA}}$ is the hepatic arterial flow fraction. Because normal sinusoids are similar in transporter and enzyme composition, the outlet blood concentrations from the different sinusoids emptying into the liver veins are, in practice, identical (1); liver kinetics can be modeled in terms of a single equivalent sinusoid.

In humans, however, the PV is inaccessible for blood sampling or direct flow measurements. Accordingly, several attempts have been made to estimate the dual-input timeactivity curve from dynamic PET data without the need for invasive blood flow or concentration measurements $(11,12)$. However, as yet, no method has successfully solved the problems of partial-volume effects of the PV, which unavoidably affect the time-activity curve because of the size of the vessel and respiratory movements. Respiration-gated recordings may help resolve some of these problems. Once fully developed, completely noninvasive methods will be attractive for use in human studies; however, until this ambitious goal has been reached, alternative methods will continue to be explored.

Various attempts have been made to calculate dual input on the exclusive basis of measurements of the arterial timeactivity curve. In a pioneering study, Choi et al. (13) applied a time delay to the arterial ${ }^{18} \mathrm{~F}$-FDG time-activity curve to account for the delay in the PV tracer input in healthy human subjects, but they did not account for dispersion. In an attempt to do so, and to keep the model as simple and physiologically correct as possible, we recently successfully applied a model of the transfer of the tracer from the intestinal arteries through the splanchnic circulation to estimate the PV time-activity curve (14). This PV model includes a single parameter $\beta(\mathrm{min})$ that accounts for both delay and dispersion through the prehepatic splanchnic circulation:

$$
\tilde{C}_{P V}(t)=\int_{0}^{t} h(t-\tau) C_{A}(\tau) d \tau
$$

where $h(t)$, the 1-parameter impulse-function, is $\beta /(t+\bar{\beta})^{2}$, $\tilde{C}_{P V}(t)$ is the model-derived PV time-activity curve, and $C_{A}(t)$ is the arterial time-activity curve. The parameter $\bar{\beta}$ is the population mean of the tracer-specific $\beta$ value; it reflects, but is not identical to, the mean transit time of the tracer through the PV-drained viscera. The PV model provided a successful description of the PV time-activity curves for all tracers tested (Figs. 2A and 2B) (6).

Next, a model-derived dual-input time-activity curve, $\tilde{C}_{d u a l}(t)$, is calculated as the flow-weighted average of $C_{A}(t)$ and $\tilde{C}_{P V}(t)(6)$ :

$$
\tilde{C}_{\text {dual }}(t)=\overline{f_{H A}} C_{A}(t)+\left(1-\overline{f_{H A}}\right) \tilde{C}_{P V}(t), \quad \text { Eq. } 3
$$

where $\overline{f_{H A}}$ is the population mean of the HA fraction of total flow, being 0.25 in pigs and humans $(6,15)$. As illustrated in Figure 2 (right panel), the experimental pig data showed that the model-derived dual-input time-activity curve agreed well with the measured dual-input time-activity curve (6). Moreover, the use of $\tilde{C}_{d u a l}(t)$ and a value of 0.25 for $\overline{f_{H A}}$ yielded blood-to-cell clearances similar to those obtained using the measured dual-input time-activity curves (16). These findings validated the model-based estimation of the dual input. The solidity of the model estimation of the dual-input timeactivity curve is promising for transfer to human studies, as discussed below in the context of estimating hepatic blood perfusion.

\section{MEASUREMENTS OF HEPATIC BLOOD PERFUSION AND METABOLISM}

\section{Blood Perfusion}

The sinusoidal blood-to-hepatocyte transfer is practically unlimited for most PET tracers because of the highly fenestrated endothelial cells, the numerous microvilli of the hepatocyte plasma membrane extending into the extended plasma volume between the endothelial cells and the hepatocytes, that is, the Space of Dissé, and the numerous high-capacity transporters on hepatocyte plasma membranes. For such tracers, the blood-to-cell clearance $\left(K_{1}\right.$; $\mathrm{mL}$ of blood $/ \mathrm{min} / \mathrm{cm}^{3}$ of liver tissue) is determined by hepatic blood perfusion $(7,9)$. Note that blood flow is given in terms of, for example, $\mathrm{mL}$ of blood/min and blood perfusion is given in $\mathrm{mL}$ of blood $/ \mathrm{min} / \mathrm{cm}^{3}$ of tissue. The $K_{1}$ for ${ }^{18} \mathrm{~F}-\mathrm{FDG}$ and $3-\mathrm{O}-{ }^{11} \mathrm{C}$-methylglucose, determined from the initial measurements only, that is, 3-min dynamic PET recordings and using a model-derived dual input (Eq. 3), can be used as a measure of hepatic blood perfusion (16). The estimated $K_{1}$ values for ${ }^{18} \mathrm{~F}-\mathrm{FDG}$ and $3-O-{ }^{11} \mathrm{C}$-methylglucose were insensitive to fairly large variations in $\bar{\beta}$ and $\overline{f_{H A}}$, and although it did tend to overestimate the perfusion, the method was able to detect small changes in hepatic blood perfusion. Taken with the similar mean HA flow fractions in pigs and humans, these findings are encouraging for the transfer of the method to human studies for estimating regional hepatic blood perfusion.

\section{Metabolic Schemes}

Glucose metabolism is usually assessed using the analog tracer ${ }^{18} \mathrm{~F}$-FDG. In most cells, the metabolism of ${ }^{18} \mathrm{~F}$-FDG essentially stops after phosphorylation to ${ }^{18}$ F-FDG-6-phosphate, that is, irreversible kinetics. Therefore, the modeling of ${ }^{18}$ F-FDG metabolism usually includes the 3 rate constants $K_{1}, k_{2}$, and $k_{3}$. Hepatocytes, however, contain glucose-6phosphatase, which dephosporylates ${ }^{18}$ FDG-6-phosphate $\left(k_{4}\right)$, that is, reversible kinetics (Fig. 3). Glucose-6-phosphate is important for the liver's role in regulating blood glucose levels. Moreover, it was shown in a study using liver tissue 


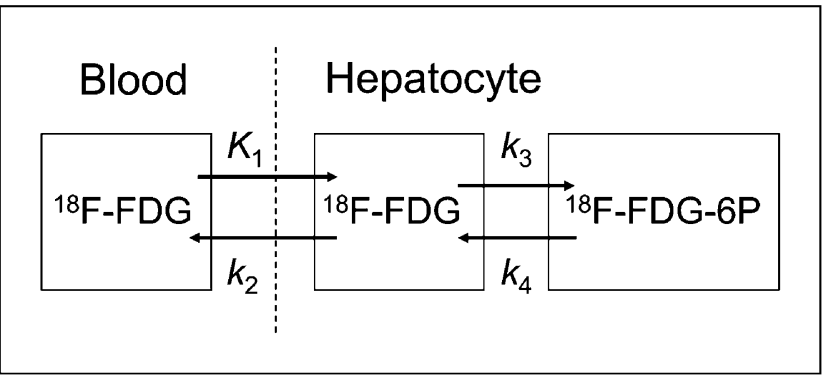

FIGURE 3. Metabolic scheme of hepatic ${ }^{18} \mathrm{~F}-\mathrm{FDG}$ metabolism. $K_{1}=$ blood-to-cell clearance ( $\mathrm{mL}$ of blood $/ \mathrm{min} / \mathrm{cm}^{3}$ of liver tissue); $k_{2}=$ rate constant for back flux of ${ }^{18} \mathrm{~F}-\mathrm{FDG}$ from cell to blood $\left(\mathrm{min}^{-1}\right) ; k_{3}=$ rate constant for intracellular enzymatic conversion of ${ }^{18} \mathrm{~F}$-FDG to ${ }^{18} \mathrm{~F}-\mathrm{FDG}$ 6-phosphate $\left(\mathrm{min}^{-1}\right)$; and $k_{4}=$ rate constant for intracellular enzymatic dephosphorylation of ${ }^{18} \mathrm{~F}$-FDG-6-phosphate to ${ }^{18} \mathrm{~F}-\mathrm{FDG}\left(\mathrm{min}^{-1}\right)$.

samples from pigs that ${ }^{18}$ F-FDG-6-phosphate is metabolized even further (17). Therefore, a more accurate model would include additional rate constants, but even the use of a $K_{1}-k_{4}$ model would underestimate $k_{4}$ because a fraction of the metabolites is not ${ }^{18} \mathrm{~F}-\mathrm{FDG}-6-\mathrm{P}$.

Hepatic lipid metabolism has been assessed using ${ }^{11} \mathrm{C}$ palmitate PET $(18,19)$. The data were analyzed by including parallel oxidation and triglyceride formation in the liver and partial release of labeled triglycerides into the circulation. The model was validated by measuring ${ }^{11} \mathrm{C}$-palmitate and ${ }^{11} \mathrm{C}$-triglyceride in the arterial, portal, and liver venous blood in pigs (18). The interpretation of the data also addressed the interaction between hepatic metabolism and the metabolism of intestinal fat. These studies clearly showed that even complex hepatic metabolism and interactions with other organs can be addressed by meticulous study designs and experimental procedures.

The hepatic metabolism of ammonia shows a sinusoidal zoning with periportal urea formation and perivenous formation of glutamine. This was quantified in a PET study in pigs using an experimental procedure with the administration of ${ }^{13} \mathrm{~N}$-ammonia into the HA and PV, respectively, and it was shown that half the ammonia was converted into urea and half into glutamine (20). Thus, the sinusoidal zoning of metabolism was successfully assessed by PET.

\section{Metabolic Clearance}

The irreversible metabolism of a tracer can be assessed through the metabolic clearance of a tracer from the blood to intracellular metabolites, $K_{\text {met }}\left(\mathrm{mL}\right.$ of blood $/ \mathrm{min} / \mathrm{cm}^{3}$ of liver tissue), when there is tracer equilibrium across the plasmacell membrane, that is, in quasi steady state. For tracers not trapped in the prehepatic splanchnic area within the measurement acquisition time, the arterial time-activity curve can be used as an input function $(7,21)$. Then, $K_{\text {met }}$ can be estimated as the asymptote fitted to the linear part of the relationship between the externally measured amount of radioactivity in the region of interest, $M(t)$, and arterial time-activity curve, $C_{A}(t)(22,23)$ :

$$
M(t) / C_{A}(t)=V+K_{\text {met }}\left[\int_{0}^{t} C_{A}(\tau) d \tau\right] / C_{A}(t), \quad \text { Eq. } 4
$$

where $V\left(\mathrm{~mL}\right.$ of blood $/ \mathrm{cm}^{3}$ of tissue $)$ is a virtual volume of distribution and $\left[\int_{0}^{t} C_{A}(\tau) d \tau\right] / C_{A}(\tau)$ is a virtual time (min). The symbols $V$ and $M(t)$ refer to the total tissue volume sampled (region of interest). At any given time point during quasi steady-state metabolism, $M(t)$ is a linear function of the amount of tracer supplied by the blood up to the time point in $\int_{0}^{t} C_{A}(\tau) d \tau$, with $K_{\text {met }}$ being the slope of the relationship at quasi steady-state metabolism. The metabolic clearance $K_{\text {met }}$ is a solid measure because $\int_{0}^{t} C_{A}(\tau) d \tau$ is not affected by minor inaccuracies in registering the starting time point of blood sampling and PET recording, in contrast to the estimation of $K_{1}$, which is based on the initial tracer distribution (7). The relationship in Equation 4 requires only irreversible quasi steady-state metabolism and is not confined to any specific kinetics model.

If the metabolic clearance $K_{\text {met }}$ (Eq. 4) is interpreted in the context of a detailed compartmental model with irreversible metabolism (e.g., Fig. 3, when $k_{4}=0$ ), the following equation applies (22):

$$
K_{\text {met }}=K_{1} \frac{k_{3}}{k_{2}+k_{3}} .
$$

This equation relates $K_{\text {met }}$ to the rate constants of metabolism, and it can be seen that if there is a back flux of tracer from cell to blood, that is, $k_{2}$ is not equal to 0 , then $K_{\text {met }}$ is less than $K_{1}(24)$. As mentioned, however, this model also requires a dual input.

The standard use of an average tissue radioactivity concentration within a given time frame (static scan), such as, for example, the standardized uptake value (i.e., radioactivity concentration in the tissue normalized to dose and body weight), includes the concentrations of the tracer and possible metabolites in both cells and blood within the organ. In contrast, $K_{\text {met }}$, being a result of the mathematic relationship in Equation 4, does not include concentrations of the tracer or metabolites. The large hepatic blood volume makes this difference between $K_{\text {met }}$ and the tissue radioactivity concentration especially apparent for the liver. In a dynamic ${ }^{18}$ F-FDG PET study on 24 patients with severe liver impairment due to primary sclerosing cholangitis who were screened for cholangiocarcinoma, images of average tissue radioactivity concentrations of static PET recordings were compared with parametric images of $K_{\text {met }}(25)$. Hot spots, that is, areas with higher radioactivity or $K_{\text {met }}$ than in the surrounding tissue, were identified on both images (Fig. 4) in 3 of the patients, and the hot spot-to-reference tissue ratios were markedly higher for $K_{\text {met }}$ than for the mean radioactivity concentration. Thus, the use of $K_{\text {met }}$ parametric images increased the sensitivity and specificity of tumor detection in the liver compared with the images of radioactivity concentrations. This finding demonstrates the merit of dynamic PET compared with static PET. 

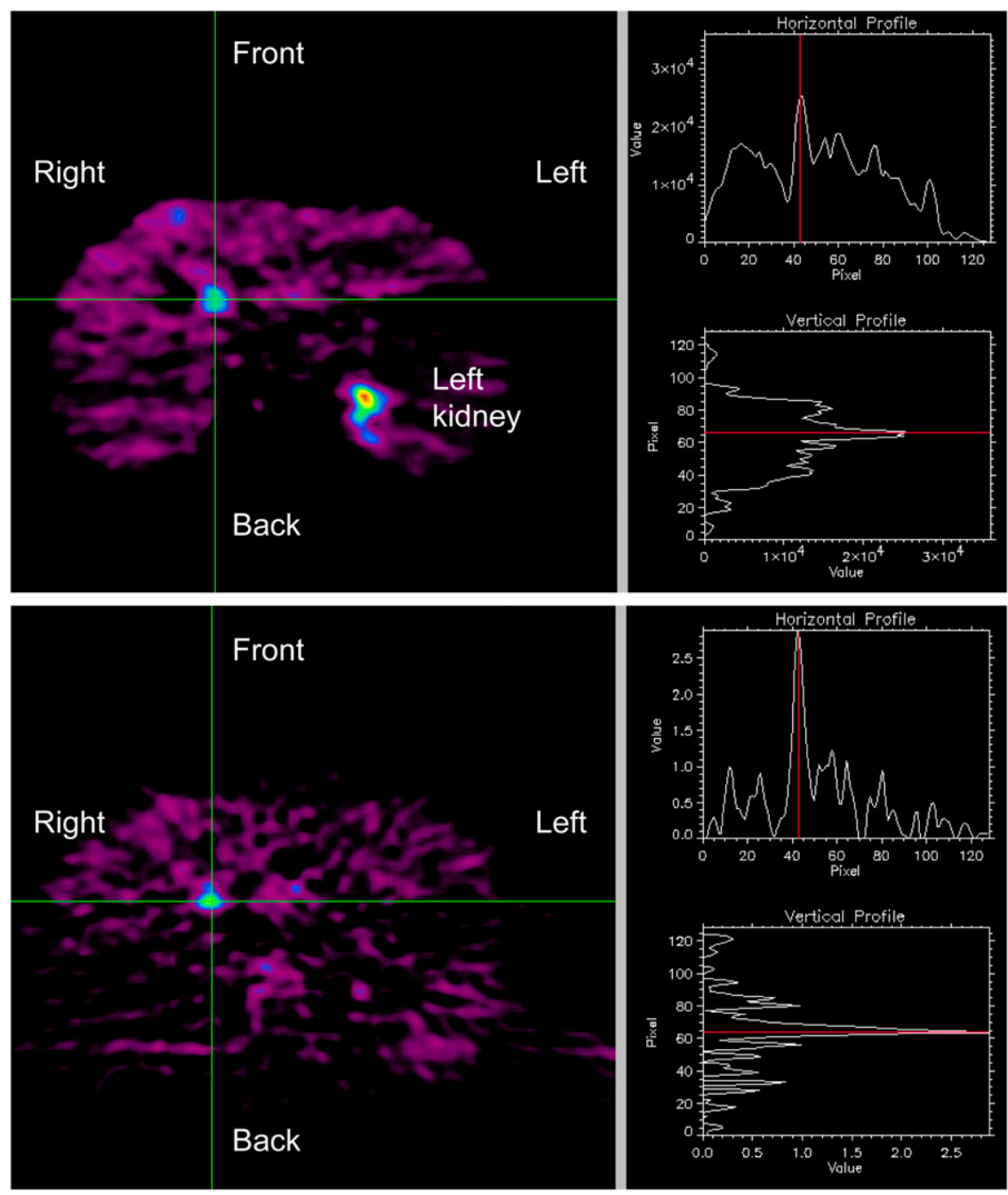

FIGURE 4. Example of ${ }^{18} \mathrm{~F}-\mathrm{FDG}$ PET images from patient with primary sclerosing cholangitis waiting for liver transplantation. (Top left) Transaxial static PET image of mean radioactivity concentrations $\left(\mathrm{kBq} / \mathrm{cm}^{3}\right.$ of liver tissue) $60-90$ min after ${ }^{18} \mathrm{~F}-\mathrm{FDG}$ injection. Green lines intersect at hot spot. (Top right) Corresponding horizontal and vertical profiles. Hot spot-to-surrounding tissue ratio was approximately 2. (Bottom left) Transaxial parametric image of $K_{\text {met }}$ of ${ }^{18} \mathrm{~F}-\mathrm{FDG}\left(\mathrm{mL}\right.$ of blood $/ \mathrm{min} / \mathrm{cm}^{3}$ of liver tissue) at same level as above. Green lines intersect at hot spot. (Bottom right) Corresponding horizontal and vertical $K_{\text {met }}$ profiles. Hot spot-to-surrounding tissue ratio was larger than 5. Explanted liver showed cholangiocarcinoma in hilar region. Study provides example of increased sensitivity of dynamic vs. static PET (Reprinted with permission of (25).)

\section{COMPARTMENTAL MODELING AND LIVER MICROCIRCULATION}

The rate constants of hepatic metabolic pathways are traditionally determined using compartmental models (Fig. 3). Intracellular tracer and possible metabolites, for example, ${ }^{18}$ F-FDG and ${ }^{18}$ F-FDG-6-P, are considered to be in separate compartments and at uniform concentrations in each compartment at each time point. The rate constants are determined by fitting a tracer-specific model to the data using tissue timeactivity curves as the output and blood time-activity curves of the tracer and possible radiolabeled metabolites as the inputs.

Compartmental models appear to be suitable for analyzing PET data because PET recordings using external detection provide data in the form of average radioactivity concentrations in regions of interest. However, compartmental models do not include tracer concentration gradients along the direction of flow in the sinusoids and capillaries, resulting from convective transport by blood perfusion, blood-cell exchange, and intracellular metabolism (Fig. 5) (26,27). In a compartmental model, the blood concentration of the tracer throughout the vascular compartment is assumed to be equal to the input concentration. In other words, the model assumes "inlet concentration equilibration" and ignores substrate concentration gradients along the vascular path. In an attempt to overcome this problem, compartmental models are sometimes combined with the Renkin-Crone relationship for first-order steady-state removal kinetics in a capillary (28):

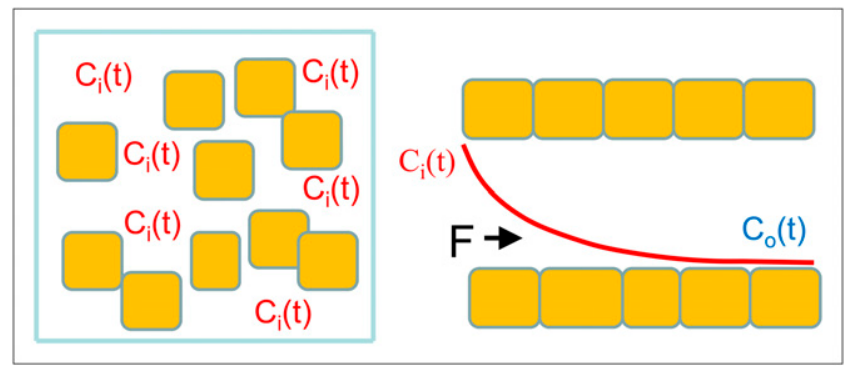

FIGURE 5. Comparison between standard compartmental model (left) and microvascular model (right). $C_{i}(t)=$ tracer concentration in input blood supply. In standard compartmental model, tracer is uniformly and instantaneously distributed throughout compartment with concentration $C_{i}(t)$. In microvascular model, here at steadystate metabolism, tracer concentration gradients develop along direction of flow in sinusoid from $C_{\mathrm{i}}(t)$ to outlet concentration $C_{\mathrm{o}}(t)$ as described previously in detail $(26,27,29,30)$. 


$$
K_{\mathrm{met}}=-F \ln \left(1-E^{*}\right)
$$

where $F$ is the blood perfusion and $E^{*}$ is the steady-state extraction fraction of the tracer. However, this modification of the standard compartmental model can lead to paradoxes, such as the kinetic rate constants being time-dependent and the tracers being cleared from a capillary more quickly than they can be supplied by the blood flow $(29,30)$. Moreover, in the standard compartmental models, a tracer that has entered the cells ( $K_{1}$ in Fig. 3$)$ and then reenters blood ( $k_{2}$ in Fig. 3) never enters the cells downstream (30). This is obviously not physiologically correct.

In vivo, when a PET tracer bolus passes through the capillaries or sinusoids, spatial and temporal concentration gradients develop along the vascular path and in the surrounding cells. Munk et al. developed models of microcirculation that account for these phenomena (30), which are of special importance for the liver because of its large blood volume. The microvascular models can be regarded as a revision of tracer input from the sinusoidal blood. The models make use of the spatial averaging of the concentrations in the sinusoidal volume, which is what the PET camera actually is recording. The microvascular models have been developed for both single- and multicapillary systems and include the effects of nonexchanging vessels. The application of the model to pig experiments using dynamic ${ }^{15} \mathrm{O}$-carbon monoxide PET yielded hepatic blood perfusion values that were comparable to independent flow meter measurements (31).

During quasi steady-state metabolism, the sinusoidal tracer concentration decreases from the sinusoidal inlet to the outlet because of convective transport in the bloodstream and substrate removal by the hepatocytes $(26,27)$. The concentration profile of an analog tracer may differ from that of the mother substance because of different enzyme affinities for the 2 substrates, as is reflected in the fact that the proportionality constant between clearances of tracer and mother substances - that is, the lumped constant $=\ln \left(1-E^{*}\right) / \ln (1-E)$ - is different from unity (32) (e.g., for ${ }^{18}$ F-FDGal and galactose $(21,33))$.

\section{HEPATIC METABOLISM STUDIED BY PET}

\section{Glucose Metabolism}

The liver plays a critical role in regulating blood glucose levels. By using dynamic ${ }^{18}$ F-FDG PET, Iozzo et al. were the first to show that physiologic hyperinsulinemia enhances hepatic glucose uptake in humans and pigs (34); in agreement with these findings, the authors visualized insulin receptors in the rat liver using ${ }^{124}$ I-labeled human insulin and PET (35). They also demonstrated an inverse relationship between hepatic glucose uptake and liver fat content (36).

$K_{1}$, determined from the initial $3 \mathrm{~min}$ of a dynamic ${ }^{18} \mathrm{~F}$ FDG PET study, can be used as a measure of hepatic blood perfusion in pigs (16). When validated in humans, an interesting clinical perspective is that a bolus injection of ${ }^{18} \mathrm{~F}-\mathrm{FDG}$ can be used to combine the measurements of hepatic blood perfusion $\left(K_{1}\right)$ and hepatic glucose metabolism $\left(K_{\text {met }}\right)$ using only the arterial time-activity curve if the PET recording is continued for 45-60 min. An interesting clinical perspective of such an adaptation is that the detection of arterialized tumor nodules in the liver could then be achieved using standard ${ }^{18} \mathrm{~F}-\mathrm{FDG}$ administration and dynamic PET recording.

\section{Fatty Acid Metabolism}

The hepatic metabolism of fatty acids has been investigated using ${ }^{11} \mathrm{C}$-palmitate as a true tracer $(18,19)$ and $14(R, S)-{ }^{18}$ F-fluoro-6-thia-heptadecanoic acid as an ana$\log$ tracer $(37,38)$. PET studies of the latter found that patients with impaired glucose tolerance had reduced hepatic uptake of fatty acids compared with healthy subjects-an example of the reciprocal hepatic regulation of these metabolic processes (38). Using dynamic ${ }^{11} \mathrm{C}$ palmitate PET/CT, Iozzo et al. showed that obese humans have increased levels of hepatic oxidation of fatty acids (19). These tracers could thus have potential for exploring the pathophysiologic effects of severe obesity on hepatic metabolism.

\section{Galactose Metabolism}

Galactose and its analog tracer ${ }^{18}$ F-FDGal comprise an interesting pair of substrates for both methodologic and clinical PET studies. Galactose is not normally present in blood, and therefore the concentration of galactose can be experimentally varied; also, galactose metabolism is independent of blood glucose, insulin, and other hormones. Galactose is almost exclusively metabolized by hepatocytes, with the first step of its metabolism being phosphorylation by galactokinase. Phosphorylated galactose is trapped in the hepatocyte, and with the exception of a minor proportion used for synthesis of phosphoglycolipids, most galactose is converted to glucose via the Leloir pathway (39). The hepatic removal of galactose follows Michaelis-Menten kinetics in vivo $(27,40)$. The hepatic galactose elimination rate at near-saturating concentration, that is, the galactose elimination capacity, is used as a measure of metabolic liver function (41), with documented prognostic information for patients with liver diseases (42). However, the galactose elimination capacity does not provide any information on possible intrahepatic regional variations that are also clinically relevant and can be explored by PET

${ }^{18} \mathrm{~F}-\mathrm{FDG}$ al is produced by a procedure similar to that used for the routine production of ${ }^{18}$ F-FDG (43). Like galactose, ${ }^{18} \mathrm{~F}$-FDGal is phosphorylated in hepatocytes by galactokinase (44). In a recent study we developed a method for estimating regional hepatic metabolic function using combined dynamic ${ }^{18} \mathrm{~F}-\mathrm{FDG}$ al PET/CT of the liver and measurements of blood concentrations of ${ }^{18} \mathrm{~F}-\mathrm{FDGal}$ in a radial artery and liver vein in healthy humans (33). The ${ }^{18} \mathrm{~F}-$ FDGal accumulates avidly in the liver, and there is no sign of significant prehepatic splanchnic ${ }^{18} \mathrm{~F}-\mathrm{FDG}$ al accumulation (Fig. 6), thus validating the use of a single arterial input 


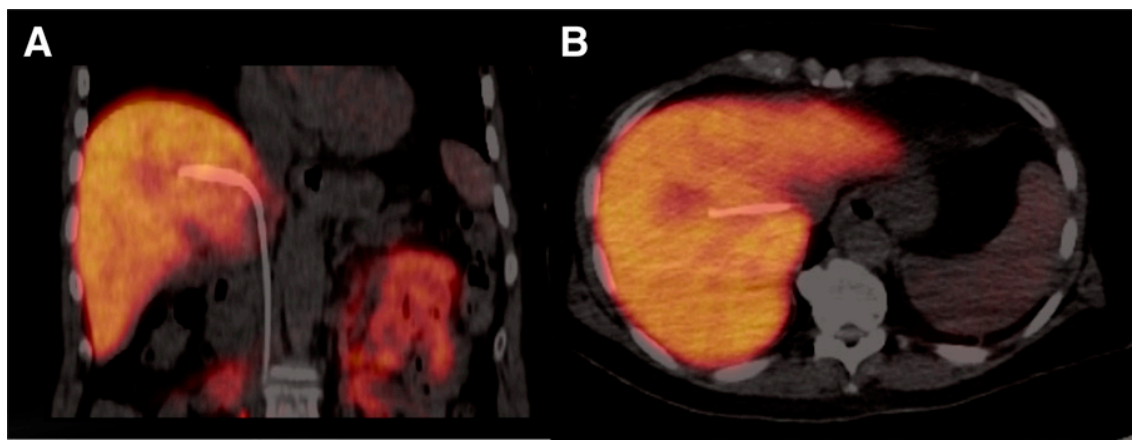

FIGURE 6. Coronal (A) and transaxial (B) ${ }^{18} \mathrm{~F}-\mathrm{FDG}$ al PET/CT images of mean tissue radioactivity concentration $\left(\mathrm{kBq} / \mathrm{cm}^{3}\right.$ of tissue) from PET study without galactose infusion in healthy human subject. Position of liver vein catheter inserted via right femoral vein can be seen. Tracer specifically accumulates in liver tissue. (Reprinted from (33).)

instead of a dual input for calculating the hepatic metabolic clearance $K_{\text {met }}$ of ${ }^{18} \mathrm{~F}-\mathrm{FDGal}$.

A comparison of PET/CT with and without simultaneous intravenous galactose infusion showed that the accumulation of radioactivity in the liver tissue and $K_{\text {met }}$ of ${ }^{18} \mathrm{~F}$ FDGal were significantly higher in the absence than in the presence of galactose (Fig. 7) (33). This finding was due to competitive inhibition of hepatic phosphorylation of ${ }^{18} \mathrm{~F}-\mathrm{FDG}$ al by galactose and verifies that ${ }^{18} \mathrm{~F}-\mathrm{FDG}$ al can be used as a tracer for hepatic galactose metabolism.

When the PET/CT study was performed without galactose infusion, that is, at true first-order kinetics, the $K_{\text {met }}$ of ${ }^{18} \mathrm{~F}$-FDGal was, on average, $85 \%$ of the intrinsic clearance of ${ }^{18} \mathrm{~F}-\mathrm{FDGal}\left(V^{*}{ }_{\max } / K_{\mathrm{m}}^{*}\right)$, which was determined by blood sample measurements (33). Accordingly, the $K_{\text {met }}$ of ${ }^{18} \mathrm{~F}$-FDGal reflects regional $V^{*}{ }_{\max }$, provided that the Michaelis constant $\left(K^{*}{ }_{\mathrm{m}}\right)$ is unaffected by changes in physiologic or pathophysiologic conditionsa reasonable assumption $(45,46)$. In contrast, for native galactose, the first-order kinetics in healthy human subjects is flow-limited and the hepatic clearance flow- determined (47). These differences are due to high and low galactokinase activity in vivo for galactose and ${ }^{18} \mathrm{~F}$ FDGal, respectively. Thus, PET-determined $K_{\text {met }}$ values of hepatic ${ }^{18} \mathrm{~F}-\mathrm{FDG} a \mathrm{l}$ metabolism can be used as a measure of regional hepatic galactokinase activity (33). These results show promise for clinical assessments of the regional variation and heterogeneity of metabolic functioning of the liver in patients with liver cirrhosis and, for example, the prognostic evaluation of metabolic liver functioning after liver resection.

Preliminary results from patients treated with stereotactic radiotherapy for liver tumors in otherwise healthy livers and examined by dynamic ${ }^{18} \mathrm{~F}$-FDGal PET/CT before and after treatment showed a significant correlation between the regional irradiation dose and the reduction in uptake of ${ }^{18} \mathrm{~F}-\mathrm{FDG}$ (48). These findings have exciting prospects for clinical use of ${ }^{18} \mathrm{~F}-$ FDGal PET/CT in planning stereotactic radiation therapy.

\section{Bile Acid Excretion}

Excretion of conjugated bile acids into bile is an essential function of the liver, and impairment of canalicular bile acid
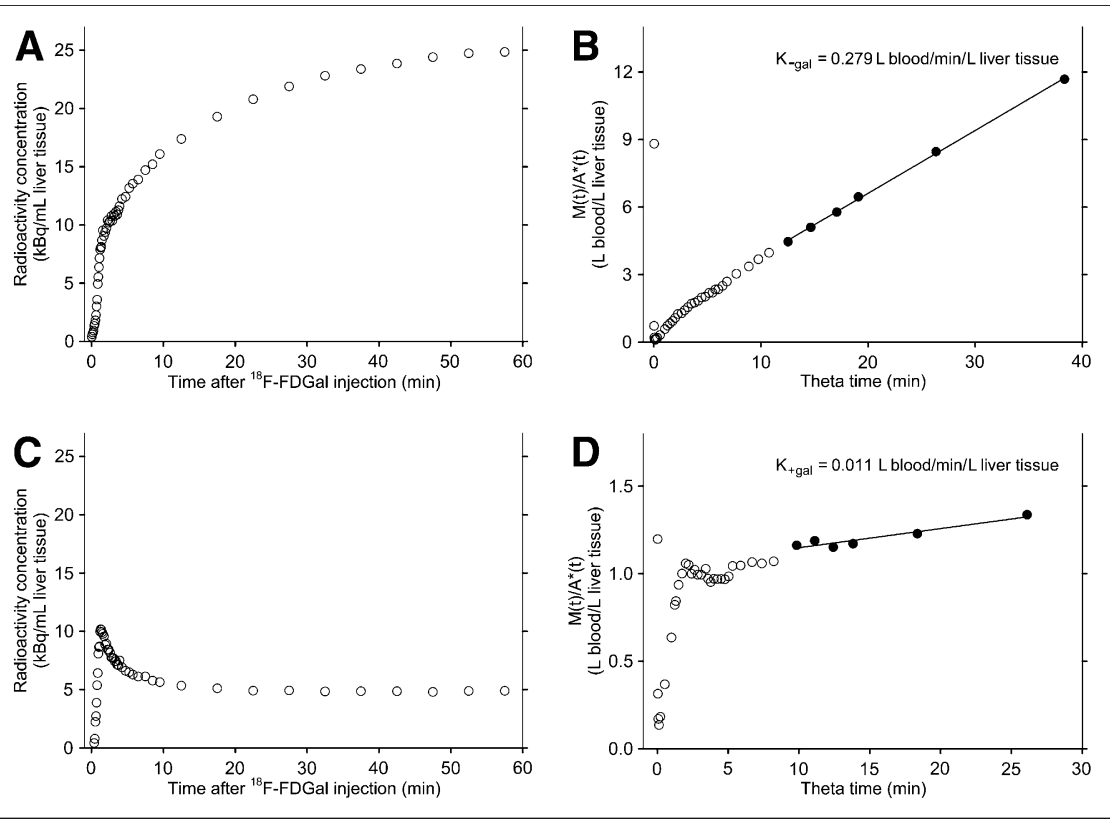

FIGURE 7. In vivo quantification of human hepatic ${ }^{18} \mathrm{~F}-\mathrm{FDG}$ al kinetics by dynamic PET/ CT studies in healthy subject without simultaneous galactose infusion (A and $B$ ) and with simultaneous galactose infusion (C and $D$ ). (A and C) Liver tissue time-activity curves. (B and D) Gjedde-Patlak plots (Eq. 4). (Reprinted from (33).) 
secretion leads to cholestatic liver injury. We recently synthesized a conjugated bile acid analog, $\left[N\right.$-methyl- $\left.{ }^{11} \mathrm{C}\right]$ cholylsarcosine, and PET studies with this analog in anesthetized pigs showed that the hepatic handling of tracer uptake from blood and excretion into the bile were comparable to that for endogenous conjugated bile acids (49). Consequently, PET/ CT with this analog may prove to be useful in humans for investigation of bile acid excretion during normal physiologic conditions and in patients with intrahepatic cholestatic liver disease or drug-induced cholestasis.

\section{CONCLUSION}

Recent developments in the use of dynamic PET for examining liver physiology and biochemistry in vivo have led to rapidly evolving methods for the direct quantification of regional blood perfusion, metabolic processes, and biliary excretory functions with clinical applications and as promising research tools for gaining a deeper insight into liver physiology and pathophysiology.

\section{ACKNOWLEDGMENTS}

This study was supported by the National Institute of Diabetes and Digestive and Kidney Diseases (R01DK074419). I thank Ludvik Bass, Ole Lajord Munk, and Michael Sørensen for their invaluable and inspiring collaboration over many years.

\section{REFERENCES}

1. Merkel C, Montagnese S, Amodio P. Functional anatomy of liver circulation. In: Keiding S, Sørensen S, eds. Functional Molecular Imaging in Hepatology. Sharjah, UAE: Bentham Science Publishers; 2012:3-13.

2. Sørensen M. Hepatocellular carcinoma. In: Keiding S, Sørensen S, eds. Functional and Molecular Imaging in Hepatology. Sharjah, UAE: Bentham Science Publishers; 2012:87-94.

3. Prytz H. Cholangiocarcinoma. In: Keiding S, Sørensen S, eds. Functional and Molecular Imaging in Hepatology. Sharjah, UAE: Bentham Science Publishers; 2012:95-103.

4. Jones-Jackson LB, Walker RC, Delbeke D. Liver metastases from colorectal cancers. In: Keiding S, Sørensen S, eds. Functional and Molecular Imaging in Hepatology. Sharjah, UAE: Bentham Science Publishers; 2012:104-119.

5. Ambrosini V, Campana D, Tommassetti P, Fanti S. Neuroendocrine tumours with secondary liver lesions. In: Keiding S, Sørensen S, eds. Functional and Molecular Imaging in Hepatology. Sharjah, UAE: Bentham Science Publishers; 2012:120-128.

6. Winterdahl M, Keiding S, Sørensen M, Mortensen FV, Alstrup AKO, Munk OL. Tracer input for kinetic modelling of liver physiology determined without sampling portal venous blood in pigs. Eur J Nucl Med Mol Imaging. 2011; 38:263-270.

7. Munk OL, Bass L, Roelsgaard K, et al. Liver kinetics of glucose analogs measured in pigs by PET: importance of dual-input blood sampling. J Nucl Med. 2001;42:795-801.

8. Brix G, Ziegler SI, Bellemann ME, et al. Quantification of $\left[{ }^{18} \mathrm{~F}\right] \mathrm{FDG}$ uptake in the normal liver using dynamic PET: impact and modeling of the dual hepatic blood supply. J Nucl Med. 2001;42:1265-1273.

9. Iozzo P, Jarvisalo MJ, Kiss J, et al. Quantification of liver glucose metabolism by positron emission tomography: validation study in pigs. Gastroenterology. 2007; 132:531-542.

10. Kudomi N, Slimani L, Järvisalo MJ, et al. Non-invasive estimation of hepatic blood perfusion from $\mathrm{H}_{2}{ }^{15} \mathrm{O}$ PET images using tissue-derived arterial and portal input functions. Eur J Nucl Med Mol Imaging. 2008;35:1899-1911.

11. Kudomi N, Jarvisalo MJ, Kiss J, et al. Non-invasive estimation of hepatic glucose uptake from $\left[{ }^{18} \mathrm{~F}\right] \mathrm{FDG}$ PET images using tissue-derived input functions. Eur J Nucl Med Mol Imaging. 2009;36:2014-2026.
12. Rani S, Kooresh S. Reconstruction of liver dual input function by optimization of the hepatic artery (HA)-to-portal vein (PV) transfer function [abstract]. $J$ Nucl Med. 2011;52(suppl):180P.

13. Choi Y, Hawkins RA, Huang SC, et al. Evaluation of the effect of glucose ingestion and kinetic model configurations of FDG in the normal liver. $\mathrm{J} \mathrm{Nucl}$ Med. 1994;35:818-823.

14. Munk OL, Keiding S, Bass L. Impulse-response function of splanchnic circulation with model-independent constraints: theory and experimental validation. $\mathrm{Am}$ J Physiol Gastrointest Liver Physiol. 2003;285:G671-G680.

15. Doi R, Inoue K, Kogire M, et al. Simultaneous measurements of hepatic arterial and portal venous flows by transit time ultrasound volume flowmetry. Surg Gynecol Obstet. 1988;167:65-69.

16. Winterdahl M, Munk OL, Sørensen M, Mortensen FV, Keiding S. Hepatic blood perfusion measured by 3-min dynamic FDG PET in pigs. J Nucl Med. 2011; 52:1119-1124.

17. Bender D, Munk OL, Feng HQ, et al. Metabolites of ${ }^{18} \mathrm{~F}-\mathrm{FDG}$ and $3-\mathrm{O}-{ }^{11} \mathrm{C}-$ methyl-glucose in pig liver. $J$ Nucl Med. 2001;42:1673-1678.

18. Guiducci L, Järvisalo M, Kiss J, et al. $\left[{ }^{11} \mathrm{C}\right]$ palmitate kinetics across the splanchnic bed in arterial, portal and hepatic venous plasma during fasting and euglycemic hyperinsulinemia. Nucl Med Biol. 2006;33:521-528.

19. Iozzo P, Bucci M, Roivainen A, et al. Liver fatty acid metabolism in the liver, measured by positron emission tomography, is increased in obese individuals. Gastroenterology. 2010;139:846-856.

20. Keiding S, Munk OL, Roelsgaard K, Bender D, Bass L. Positron emission tomography of hepatic first-pass metabolism of ammonia in pig. Eur $\mathrm{J} \mathrm{Nucl}$ Med. 2001;28:1770-1775.

21. Sørensen M, Munk OL, Mortensen FV, et al. Hepatic uptake and metabolism of galactose can be quantified in vivo by $2-\left[{ }^{18} \mathrm{~F}\right]$ fluoro-2-deoxy-galactose positron emission tomography. Am J Physiol Gastrointest Liver Physiol. 2008;295: G27-G36.

22. Gjedde A. Calculation of cerebral glucose phosphorylation from brain uptake of glucose analogs in vivo: a re-examination. Brain Res. 1982;257:237-274.

23. Patlak CS, Blasberg RG, Fenstermacher JD. Graphical evaluation of blood-tobrain transfer constants from multiple-time uptake data. J Cereb Blood Flow Metab. 1983;3:1-7.

24. Sørensen M, Munk OL, Keiding S. Backflux of ammonia from brain to blood in human subjects with and without hepatic encephalopathy. Metab Brain Dis. 2009;24:237-242.

25. Prytz H, Keiding S, Björnsson E, et al. Dynamic FDG PET is useful for detection of cholangiocarcinoma in patients with PSC listed for liver transplantation. Hepatology. 2006;44:1572-1580.

26. Bass L, Keiding S, Winkler K, Tygstrup N. Enzymatic elimination of substrates flowing through the intact liver. J Theor Biol. 1976;61:393-409.

27. Keiding S, Johansen S, Winkler K, Tønnesen K, Tygstrup N. Michaelis-Menten kinetics of galactose elimination by the isolated perfused pig liver. Am J Physiol. 1976;230:1302-1313.

28. Crone $\mathrm{C}$. The permeability of capillaries in various organs as determined by the use of the 'indicator diffusion' method. Acta Physiol Scand. 1963;58:292-305.

29. Munk OL, Keiding S, Bass L. Combining compartmental and microvascular models in interpreting dynamic PET data. In: Keiding S, Sørensen S, eds. Functional and Molecular Imaging in Hepatology. Sharjah, UAE: Bentham Science Publishers; 2012:24-31.

30. Munk OL, Keiding S, Bass L. Capillaries within compartments: microvascular interpretation of dynamic positron emission tomography data. J Theor Biol. 2003;225:127-141.

31. Munk OL, Bass L, Feng H, et al. Determination of regional flow by use of intravascular PET tracers: microvascular theory and experimental validation for pig livers. J Nucl Med. 2003;44:1862-1870.

32. Bass L, Sørensen M, Munk OL, Keiding S. Analogue tracers and lumped constant in capillary beds. J Theor Biol. 2011;285:177-181.

33. Sørensen M, Mikkelsen KS, Frisch K, Bass L, Bibby BM, Keiding S. Hepatic galactose metabolism quantified in humans using 2-[ $\left[{ }^{18} \mathrm{~F}\right]$ fluoro-2-deoxy-Dgalactose PET/CT. J Nucl Med. 2011;52:1566-1572.

34. Iozzo P, Geisler F, Oikonen V, et al. Insulin stimulates liver glucose uptake in humans: an ${ }^{18}$ F-FDG PET study. J Nucl Med. 2003;44:682-689.

35. Iozzo P, Osman S, Glaser M, et al. In vivo imaging of insulin receptors by PET: preclinical evaluation of iodine-125 and iodine-124 labelled human insulin. Nucl Med Biol. 2002;29:73-82.

36. Borra R, Lautamäki R, Parkkola R, et al. Inverse association between liver fat content and hepatic glucose uptake in patients with type 2 diabetes mellitus. Metabolism. 2008;57:1445-1451.

37. Iozzo P, Turpeinen AK, Takala T, et al. Liver uptake of free fatty acids in vivo in humans as determined with $14(\mathrm{R}, \mathrm{S})-\left[{ }^{18} \mathrm{~F}\right]$ fluoro-6-thia-heptadecanoic acid and PET. Eur J Nucl Med Mol Imaging. 2003;30:1160-1164. 
38. Iozzo P, Turpeinen AK, Takala T, et al. Defective liver disposal of free fatty acids in patients with impaired glucose tolerance. J Clin Endocrinol Metab. 2004;89:3496-3502.

39. Holden HM, Rayment I, Thoden JB. Structure and function of enzymes of the Leloir pathway for galactose metabolism. J Biol Chem. 2003;278:43885-43888.

40. Keiding S, Johansen S, Winkler K. Hepatic galactose elimination kinetics in the intact pig liver. Scand J Clin Lab Invest. 1982;42:253-259.

41. Tygstrup N. The galactose elimination capacity in control subjects and in patients with cirrhosis of the liver. Acta Med Scand. 1964;175:281-289.

42. Jepsen P, Vilstrup H, Ott P, Keiding S, Andersen PK, Tygstrup N. The galactose elimination capacity and mortality in 781 Danish patients with newly-diagnosed liver cirrhosis: a cohort study. BMC Gastroenterology. 2009;9:50-56.

43. Frisch K, Bender D, Keiding S, Sørensen M. Nucleophilic radiosynthesis of 2$\left[{ }^{18} \mathrm{~F}\right]$ fluoro-2-deoxy-D-galactose from Talose triflate and biodistribution in a porcine model. Nucl Med Biol. 2011;38:477-483.

44. Grün BR, Berger U, Oberdorfer F, et al. Metabolism and actions of 2-deoxy-2fluoro-D-galactose in vivo. Eur J Biochem. 1990;190:11-19.
45. Winkler K, Bass L, Keiding S, Tygstrup N. The physiologic basis of clearance measurements in hepatology. Scand J Gastroenterol. 1979;14:439-448.

46. Keiding S, Sørensen M. Hepatic removal kinetics: importance for quantitative measurements of liver function. In: Rodes J, Benhamou J-P, Dufour JF, Blei A, Reichen J, Rizzetto M, eds. Textbook of Hepatology: From Basic Science to Clinical Practice. 3rd ed. Oxford, UK: Blackwell; 2007: $468-478$.

47. Keiding S. Galactose clearance and liver blood flow. Gastroenterology. 1988; 94:477-481.

48. Høyer M, Sørensen M, Petersen J, Holt MI, Muren LP, Keiding S. Normal tissue reaction of the liver after stereotactic radiotherapy (SBRT) determined by ${ }^{18} \mathrm{~F}$ fluoro-deoxy-galactose and PET/CT scanning [abstract]. Radiother Oncol. 2011;99(suppl 1):S67.

49. Frisch K, Jakobsen S, Sørensen M, et al. $\left[N\right.$-methyl- $\left.{ }^{11} \mathrm{C}\right]$ cholylsarcosine, a novel bile acid tracer for PET/CT of hepatic excretory function: radiosynthesis and proof-of-concept studies in pigs. J Nucl Med. 2012. In press. 